\title{
Physical exercise and oxidative stress in muscular dystrophies: is there a good balance? Exercise and oxidative stress in MDs
}

\author{
L. CHICO, G. RICCI, M. COSCI O DI COSCIO, C. SIMONCINI, G. SICILIANO
}

Department of Clinical and Experimental Medicine, Neurological Clinic, University of Pisa, Italy.

\begin{abstract}
A B S T R A C T
The effect of oxidative stress on muscle damage inducted by physical exercise is widely debated. It is generally agreed that endurance and intense exercise can increase oxidative stress and generate changes in antioxidant power inducing muscle damage; however, regular and moderate exercise can be beneficial for the health improving the antioxidant defense mechanisms in the majority of cases. Growing evidences suggest that an increased oxidative/nitrosative stress is involved in the pathogenesis of several muscular dystrophies (MDs). Notably, physical training has been considered useful for patients with these disorders. This review will focus on the involvement of oxidative stress in MDs and on the possible effects of physical activities to decrease oxidative damage and improve motor functions in MDs patients.
\end{abstract}

Key words

Oxidative stress $\bullet$ Antioxidants $\bullet$ Regular and moderate exercise $\bullet$ Muscular dystrophies

\section{Introduction}

Muscular dystrophies (MDs) are genetic diseases characterized by progressive skeletal muscle weakness and degeneration. There are many kinds of MDs with a wide variability in term of age at onset and degree of disability (Davies and Nowak, 2006), due to mutations in different genes. The mechanisms underlying the skeletal muscle failure are complex and involve several biological pathways, including oxidative stress. Oxidative stress is a disturbance in the balance between the production of reactive oxygen/nitrogen species (ROS/RNS) and the ability to detoxify them through the antioxidant defenses (Pisoschi et al., 2015). The effect of oxidative stress on muscle damage inducted by physical exercise is widely debated. To date, physical exercise is considered to be a major component of a healthy lifestyle since it prevents several chronic diseases such as osteoporosis, diabetes, cancer, hypertension, obesity, depression, and cardiovascular disease
(Gomez-Cabrera et al., 2008). Nevertheless, the relationship between exercise and oxidative stress is extremely complex, depending on the mode, intensity, and duration of exercise. While an acute and strenuous exercise can generate an excess of free radicals production, a regular and moderate exercise seems to counteract oxidative stressrelated damage. These conflicting effects may be explained by the hormesis theory, according to which a substance, that is detrimental at high doses, induces an adaptive response that carriers out beneficial effects on the cells (Boccatonda et al., 2016). However, despite these evidences, the topic of exercise-induced oxidative stress is still unclear. In this review we will discuss: (i) the mechanisms of ROS/RNS production in the skeletal muscle and the involvement of oxidative stress in muscular dystrophies (MDs), focusing on Duchenne/Becker muscular dystrophy, myotonic dystrophy type 1, facioscapolohumeral muscular dystrophy and limbgirdle muscular dystrophies, (ii) if exercise training can be helpful to reduce oxidative stress and fatigue in MDs. 


\section{ROS/RNS production in skeletal muscle}

ROS/RNS include molecules such as superoxide anion $\left(\mathrm{O}_{2}^{-}\right)$, hydrogen peroxide $\left(\mathrm{H}_{2} \mathrm{O}_{2}\right)$, hydroxyl radicals $(\mathrm{OH} \cdot)$, nitric oxide (NO$\cdot)$, nitrogen dioxide $\left(\mathrm{NO}_{2}\right)$ and peroxynitrite $\left(\mathrm{ONOO}^{-}\right)$(Giorgio et al., 2007; Schieber and Chandel 2014; Kurutas, 2016).

Primary sources of ROS in muscle cells are, the electron transport chain (ETC) in the mitochondria, the NADPH oxidase (Nox), the xanthine oxidase (XO) and the phospholipase A2 (PLA2) (Halliwell 2007; Pwers and Jackson 2008; Steinbacher and Eckl 2015) (Figure 1).

ROS production across the ETC is a consequence of the inefficiencies in the transfer of electrons among its complexes (primarily complex I and complex III): when electrons escape from the complexes before the reduction of molecular oxygen $\left(\mathrm{O}_{2}\right)$ to water $\left(\mathrm{H}_{2} \mathrm{O}\right.$ ), they react with $\mathrm{O}_{2}$ forming $\mathrm{O}_{2}^{-}$(Brand et al., 2010). ROS production in the mitochondria of muscle cells is approximately $0.15 \%$, smaller than expected (1\%-4\%) (Steinbacher and Eckl, 2015). In a recent study, Goncalves and co-workers (2015) use new approaches (an ex vivo system) to evaluate, from muscle tissue, different production sites of $\mathrm{O}_{2}{ }^{-} / \mathrm{H}_{2} \mathrm{O}_{2}$ in the mitochondrion during rest, mild, and intense aerobic exercise. The authors provide the first evidence that not only complex I (site IF) and complex III (site IIIQo), but also sites IQ and IIF (in complex II) should be considered for ROS production. In particular, at rest $\mathrm{O}_{2}^{-}$and $\mathrm{H}_{2} \mathrm{O}_{2}$ are predominantly produced from IQ and IIF sites, followed by sites IF and IIIQo. Under conditions that mimic mild and intense aerobic exercise, total ROS production is reduced.

To a greater extent than mitochondria, Nox enzymes contribute to cytosolic $\mathrm{O}_{2}^{-}$production in skeletal muscle both at rest and during contractile activity. Nox include a family of protein consisting of seven members: Nox1-Nox5, Duox1-Duox-2 each of which shows distinct tissue-specific expression patterns (e.g. Nox1 is expressed in the smooth muscle, while Nox 2 is expressed in skeletal muscle, and neurons) (Pendyala and Natarajan, 2010).

$\mathrm{XO}$ can be a large source of free radicals production since it utilizes hypoxanthine or xanthine as substrate and $\mathrm{O}_{2}$ as cofactor to produce $\mathrm{O}_{2}{ }^{-}$and uric acid (Chung et al., 1997; Ryan et al., 2011). Upon contraction, XO activity is significantly increased leading to oxidative stress and muscle damage (Steinbacher and Eckl, 2015).
PLA2 contributes to elevation of ROS in skeletal muscle by catalyzing production of arachidonic acid by ROS-producing lipoxygenases, promoting the translocation of Nox to the sarcolemma, and increasing ROS production in mitochondria (Choi et al., 2016).

Between RNS, NO is a low-reactive molecule involved in several cellular mechanisms such as the regulation of the vasomotor tone and of immunomodulatory signaling pathways (Mangge et al., 2014) and neuronal activity (Pacher et al., 2007). NO is rapidly removed by the conversion to nitrite/nitrate, limiting its biological half-life (Pacher et al., 2007). Only in presence of $\mathrm{O}_{2}^{-}$, NO can becomes toxic forming the much more powerful oxidant $\mathrm{ONOO}^{-}$(Pacher et al., 2007; Mangge et al., 2014). NO. is synthesized by the nitric oxide synthase (NOS) enzyme that converts L-arginine into L-citrulline, utilizing NADPH and $\mathrm{O}_{2}$ as co-factors (Choi et al., 2016). NOSs are enzymes that includes different isoforms: neuronal NOS (nNOS or NOS1), inducible NOS (iNOS or NOS2) and endothelial NOS (eNOS or NOS3) (Powers and Jackson, 2008). In skeletal muscle nNOS, localized both in cytoplasm and sarcolemma, is considered the primary source of RNS. NO- is mainly synthesizes by nNOS splice variant mu $(\mathrm{nNOS} \mu)$. The $\mathrm{nNOS} \mu$ localization and activity are regulated by interaction between proteins as well as by translational modifications. $\mathrm{nNOS} \mu$ and NO. regulate hypertrophy, fiber type, force production (excitation-contraction coupling), fatigue resistance, microtubule organization, regulation of blood flow, myocyte differentiation, respiration, and glucose homeostasis (Stamler and Meissner, 2001), but the mechanisms underlying these functions are still unknown. NO activates soluble guanylyl cyclases (GCs) generating guanosine 3',5'-cyclic monophosphate (cGMP); intracellular increase of the cGMP levels may further activate cGMP-dependent protein kinase (PKG) (Wang and Robinson, 1997). In mouse models, nNOS activity can be significantly increased by regular physical exercise and aerobic training activating the NO/GCs/cGMP pathway. Conversely, down-regulation of NO production increases exercise metabolic cost and decreases running performance (Chalimoniuk et al., 2015).

Increased ROS/RNS levels induce damage to nucleic acids, lipids, and proteins that can leads to 


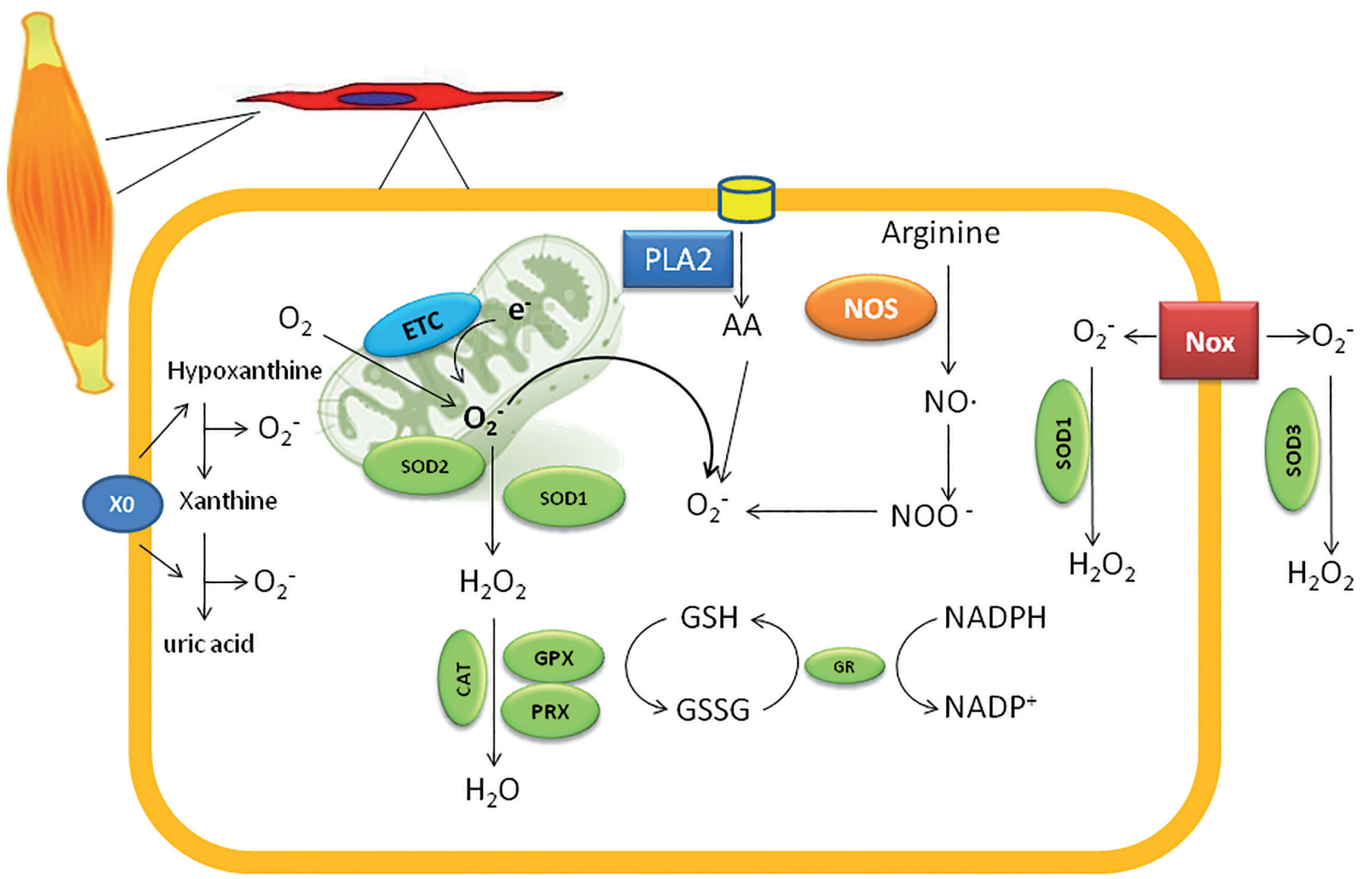

Fig. 1. - Primary sources of ROS in muscle cells. Abbreviations. ETC: electron transport chain Nox: NADPH oxidase, XO: xanthine oxidase, PLA2: phospholipase $\mathrm{A} 2, \mathrm{O}_{2}^{-}$: superoxide anion, $\mathrm{H}_{2} \mathrm{O}_{2}$ : hydrogen peroxide, $\mathrm{OH} \cdot$ : hydroxyl radicals, NO: nitric oxide, ONOO-: peroxynitrite, SOD: superoxide dismutase, CAT: catalase, GSH: glutathione, GR: glutathione reductase, GPX: glutathione peroxidase, $\mathrm{O}_{2}$ : oxygen, $\mathrm{H}_{2} \mathrm{O}$ : water, $\mathrm{GSH}$ : reduced glutathione, GSSG: oxidized glutathione, PRX: peroxiredoxina, AA: arachidonic acid.

cellular death by apoptotic mechanisms. Much of the evidences in this field have been made possible analyzing changes in several oxidative stress markers. In fact, lipid peroxidation leads to alterations in the degree of membranes fluidity, deficit of membranebound receptors and enzymes; moreover it may contribute to amplify cellular damage resulting from generation of oxidized products. Several products of lipid peroxidation are usually used as biomarkers of oxidative/nitrosative stress, such as malondialdehyde (MDA), 4-hydroxy-2-nonenal (HNE), 2-propenal, isoprostanes (F2-IsoPs) and thiobarbituric acid reactive substances (TBARS) (Kurutas, 2016; Cipak Gasparovic et al., 2016).

$\mathrm{NOO}^{-}$causes post-translational modifications of proteins, inducing nitrative stress which results in an increase of tyrosine nitration (3-nitrotyrosine, 3-NT) levels. Other biomarkers for protein-oxidative damage include: protein carbonyls, advanced lipoxidation end products, advanced glycation end products (AGEs), oxidized low-density lipoprotein (oxLDL), thought the utility of this biomarker has been criticized (Frijhoff et al., 2015), and advanced oxidation protein products (AOPP) (Bouzid et al., 2015).

It is also found that ROS induce damage to DNA and RNA; the two major biomarkers used to test any alterations in these biomolecules are 7,8-dihydro-8oxo-2c-deoxyguanosine (8oxodG) and 7,8-dihydro8-oxoguanosine (8oxoGuo), respectively (Frijhoff et al., 2015).

\section{Antioxidant compounds}

Humans have developed complex antioxidant systems, both enzymatic and non-enzymatic, which act in synergy to prevent or reduce the oxidative damage (Kurutas, 2016). Among the enzymatic molecules, play a primary role, superoxide dismutase (SOD), that exist in three isoforms (SOD1- SOD3), catalase (CAT), glutathione peroxidase (with five isoforms 
GPX1-GPX5) and glutathione reductase (GR). SOD dismutates $\mathrm{O}_{2}^{-}$to form $\mathrm{H}_{2} \mathrm{O}_{2}$ and oxygen $\left(\mathrm{O}_{2}\right)$, CAT catalyses conversion of $\mathrm{H}_{2} \mathrm{O}_{2}$ into $\mathrm{O}_{2}$ and water $\left(\mathrm{H}_{2} \mathrm{O}\right)$. GPXs reduce $\mathrm{H}_{2} \mathrm{O}_{2}$ or organic hydroperoxide ( $\mathrm{ROOH})$ into $\mathrm{H}_{2} \mathrm{O}$ and alcohol $(\mathrm{ROH})$, respectively, using reduced glutathione (GSH). This one can be oxidized to glutathione disulfide (GSSG) that can be reduced again to GSH by GR enzyme.

In addition to these antioxidants, there are other enzymes, such as thioredoxin, thioredoxin reductase, glutaredoxin, and peroxiredoxin that can be useful to maintain the redox balance (Powers and Jackson 2008) (Figure 1).

Between endogenous non-enzymatic antioxidants play a major role the thiol compounds like glutathione, lipoic acid and thioredoxins, which contain a sulphydryl group important to scavenger ROS. Also other proteins like ferritin, transferrin, ceruloplasmin, albuminuric acid, and coenzyme Q (CoQ10), are part of this "class" of antioxidants (Pisoschi et al., 2015).

However, under certain conditions that sustain ROS/ RNS production, endogenous antioxidants may not be sufficient to limit oxidative damage, and the addition of dietary antioxidants, such as vitamin $\mathrm{C}$, vitamin E, melatonin, carotenoids, and flavonoids may be used to maintain optimal cellular functions (Pisoschi et al., 2015; Kurutas, 2016).

A reduction of antioxidant enzymes (SOD, CAT, GPX, GR) activity and of GSH/GSSG ratio was found in oxidative stress conditions. Also several antioxidant capacity assays have been used among which Trolox Equivalent Antioxidant Capacity (TEAC), Total Antioxidant Status (TAS), Ferric Reducing Ability of Plasma (FRAP), Total Radical Trapping Antioxidant Parameter (TRAP), and Oxygen Radical Absorbance Capacity (ORAC) (Powers and Jackson, 2008; Fisher-Wellman and Bloomer 2009).

\section{Evidences of oxidative stress involvement in MDs}

\section{Oxidative stress in Duchenne muscular dystrophy and Becker muscular dystrophy}

Among MDs, Duchenne muscular dystrophy (DMD) is the most frequent disorder in children; it is a severe and progressive disease that affects approximately $1 / 3500$ male births. Becker muscular dystrophy (BMD) is a milder variant of DMD in which symptoms appearing later in childhood or in adolescence (Darras et al., 2000; Dahlqvist and Vissing 2016). DMD and BMD are caused by mutations in the dystrophin gene that encodes for a protein which is a member of the dystrophinassociated glycoprotein (DAG) complex. It connects the contractile components within the myofiber to the extracellular matrix and helps to stabilize the sarcolemma (Ervasti and Campbell, 1991). Patients with DMD have a complete loss of dystrophin, while patients with BMD show a reduction of dystrophin at muscle level (Dahlqvist and Vissing, 2016).

The $m d x$ mouse that harbors a point mutation in the dystrophin gene has been widely studied as a mouse model for DMD. Numerous evidences, both in $m d x$ mouse and humans, underline the involvement of oxidative stress in the pathogenesis of DMD. The absence of dystrophin appears to render muscle more susceptible to oxidative damage. In that regard, using myotube cultures derived from normal and $m d x$ mice, Rando et al. (1998) examined the susceptibility of the cells to different stressors, both pro-oxidants and non-oxidants, at different concentrations. The first compounds included $\mathrm{H}_{2} \mathrm{O}_{2}$, manadione (an inductor of several reactive species), and paraquat (it generates $\mathrm{O}_{2}^{-}$). Non-oxidant stressors used were staurosporine (a pro-apoptotic compound), A23187 (an activator of proteases and an inducer of $\mathrm{Ca}^{2+}$ ), carbonyl cyanide m-chlorophenylhydrazone (an inhibitor of mitochondrial respiration). Cells derived from $m d x$ muscles were more susceptible to pro-oxidants than normal myoblasts, but both cells populations were equally susceptible to non-antioxidant stressors. Probably, these responses were relative to dystrophin expression; indeed, undifferentiated myoblasts, that lacked in dystrophin, derived both from $m d x$ and normal mice were equally sensitive to pro-oxidants supporting the hypothesis that oxidative stress may lead to myofiber death in DMD.

Moreover, studies showed significantly higher TBARS content, while SOD1 activity was decreased, while SOD2 and CAT activities were elevated in $m d x$ mice muscles with respect to controls (Ragusa et al., 1997), and ectopic expression of CAT in mitochondria increased lifespan and led to a partial restoration of $m d x$ muscle function (Selsby, 2011). Similar results have been found in humans in 
which the levels of TBAR and the CAT and GR activities were significantly higher in muscles from DMD patients versus control subjects, while SOD activity was not altered in dystrophic muscles (Kar and Pearson, 1979). Also 8-OHdG was found to be elevated in DMD patients versus healthy controls (Rodriguez and Tarnopolsky, 2003).

The exact contribution of NO. in DMD pathogenesis is still unclear. This "gray zone" results from the secondary loss of the complex of proteins that interact with dystrophin, the so called dystrophinassociated protein complex (DPC). Considering that nNOS is a member of the DPC, it is possible that the loss of nNOS from muscle cells contributes to the dystrophic pathology (Wehling et al., 2001). A lower NO production in dystrophic muscle could have harmful effects on muscle function because NO is involved in regulating several key processes in skeletal muscle (Tidball and Wehling-Henricks, 2004). Grazdenovic et al. (1997) found (by enzyme immunochemistry and immunohistochemistry) that in DPC not only dystrophin, but also nNOS was absent in biopsies from DMD patients. In muscle tissue of BMD patients, similarly to dystrophin, nNOS expression was only reduced. Afterwards, Gücüyener and coworkers (Gücüyener et al., 2000) found that serum NOx levels were significantly lower in DMD than those of the controls. Nevertheless, other investigators observed that prenecrotic $m d x$ muscle fibers did not exhibit changes in $\cdot \mathrm{NO}$-induced nitrotyrosine formation. Moreover, NOS-null mice did not develop dystrophic symptoms, and neither NOS-null nor $m d x$ mice with ectopic NOS expression show any alteration in oxidative stress susceptibility (Rando et al., 2001). As reviewed by Heydemann and McNally (2009) nNOS null mice showed no feature of the MyDs, such as muscle degeneration. Moreover, the phenotype of nNOS and dystrophin double knockout mice was similar to that observed in the $m d x$ model.

\section{Oxidative stress in myotonic dystrophies}

Myotonic dystrophy (MyD) is the most common form of muscular dystrophy that begins in adulthood, although there is a variant, called congenital $\mathrm{MyD}$, that is apparent at birth. It is multisystemic disease, transmitted as autosomal dominant trait, characterized by a involvement of skeletal muscle and heart, but also eye, brain and endocrine system.
The most frequent form of MyD, the type 1 (DM1), is characterized by nucleotide triplet expansions [(CTG)n] in the 3'-untranslated region of the myotonic dystrophy protein kinase (DMPK) gene, underlying to the complexity and multisystemic phenotypic variants of disease (Pantic et al., 2013). The increased levels of ROS as well as a reduction of antioxidants is well documented in DM1, oxidative stress may be associated with muscular and extramuscular signs of the disease (Nikolić-Kokić et al., 2016). Previous in vitro studies (Usuki and Ishiura, 1998) suggested the involvement of oxidative stress in the disease pathogenesis. For example, the effects of oxidative stress, inducted by methylmercury, on myogenic cells with expanded CTG repeats in the myotonin protein kinase (MtPK) gene were investigated. Mutant MtPK cDNA transformants (from 46 to $160 \mathrm{CTG}$ repeats) treated with methylmercury ( $1 \mu \mathrm{M}$ for $24 \mathrm{~h}$ ) showed cell death, while in wild type MtPK cDNA transformants the toxicity and cell death were reduced In mutant transformants, the administration of N-acetyl-Lcysteine and trolox (two antioxidants) suppressed the injury inducted by methylmercury. These findings emphasized the key role of ROS to evoke cellular damage which was amplified by expanded CTG repeats in MtPK.

Also human studies have been conducted to analyze the role of oxidative stress in DM1. In our study (Siciliano et al., 2005) we reported that plasma AOPP levels, total serum g-glutamyltransferase (GGT) activity (a crucial enzyme in modulating GSH), and GGT activity associated with low-density lipoprotein were significantly increased in DM1 patients $(\mathrm{N}=38)$ with respect to controls $(\mathrm{N}=10)$. Moreover, raised AOPP levels correlated with extramuscular signs of the disease (cataracts and heart) but not with muscular involvement. Kumar et al. (Kumar et al., 2014) found significantly higher levels of MDA, and lower levels of GPX, GST and GSH in DM1 patients $(\mathrm{N}=20)$ with respect to control subjects $(\mathrm{N}=40)$, although SOD and TAS levels were increased in DM1 patients. In another recent study (Nikolić-Kokić et al., 2016), the activities of erythrocyte SOD and CAT were reduced in DM1 patients $(\mathrm{N}=30)$ versus controls $(\mathrm{N}=15)$. Moreover, a positive correlation was found between disease duration and muscular impairment rating scale score as well as with GR activity suggesting that 
reductions in antioxidant enzyme activities during DM1 disease progression trigger oxidative stress and metabolic abnormalities that could contribute to muscle atrophy.

\section{Oxidative stress in facioscapulohumeral muscular dystrophy}

Facioscapulohumeral muscular dystrophy (FSHD), the third most prevalent hereditary myopathy in adulthood, is characterized by progressive, asymmetric atrophy and weakness of a selective muscle groups. Age of onset is variable, but in the classical form the majority of patients become symptomatic in the second-third decade. FSHD has been associated with a reduced number of $3.3 \mathrm{~kb}$ repeat units, called D4Z4, localized on chromosome 4q35 (Ricci et al., 2013). A wide inter- and intrafamilial variability of clinical expression is well documented, without a clear correlation between the clinical phenotype and molecular variations (Ricci et al., 2013). To date, despite the identification of the molecular defect, its pathologic effects remain largely unknown (Ricci et al., 2014) Epigenetic mechanisms and/or loci modifications are supposed to be involved in modulating the incomplete penetrance and in influencing the disease expression (Lemmers et al., 2015). It has been proposed that the reduction of the D4Z4 array results in the transcription of a doublehomeobox transcription factor, DUX4, encoded by an open reading frame in D4Z4 repeat. Although DUX4 mRNAs and proteins are extremely low, it has been observed that the DUX4-expressing FSHD muscle nuclei show pathologic features consistent with DUX4 induced toxicity (Gatica and Rosa, 2016). According to the current pathogenic model of FSHD, epigenetic modifications, either related to the contraction of the array or to its hypomethylation, may result in the occasional escape from repression in muscle cells with a consequently inappropriate expression of DUX4 protein (Ricci et al., 2014). The overexpression of DUX4 is supposed to induce a gene deregulation cascade, including a deregulation in the oxidative stress response. The study of Turki et al. (2012) reported in muscle specimen of FSHD patients a higher oxidative stress damage, in term of lipid peroxidation, protein carbonylation and lipofuscin accumulation, in association with an abnormal mitochondrial function, decreased cytochrome $\mathrm{c}$ oxidase activity and reduced ATP synthesis. Recently, Dmitriev and coworkers (2016) provided evidence of oxidative DNA damage in vitro study using myoblasts isolated from FSHD patients. The authors reported that the addition of tempol, a powerful antioxidant, to the proliferating DUX4-transfected myoblasts and FSHD myoblasts, reduced the level of DNA damage. Notably, antioxidant treatment during the myogenic differentiation of FSHD myoblasts, significantly reduced morphological defects in myotube formation.

\section{Oxidative stress in limb-girdle muscular dystrophies}

Another group of MyDs clinically similar to DMD/ BMD is represented by the limb-girdle muscular dystrophies (LGMD). LGMD is a descriptive term that comprises different diseases with variable clinical expression, ranging from severe to mild forms, transmitted as an autosomal recessive or dominant trait (Darras et al., 2000), beginning in childhood or adulthood. The various forms of LGMD are caused by mutations in different genes that encode a wide variety of proteins, including components of the DAG complex, cytoplasmic enzymes, membrane and nuclear matrix proteins. The pathogenesis of LGMD involves different mechanisms such as increment of intracellular $\mathrm{Ca}^{2+}$ ions, infiltration of muscle tissue by inflammatory immune cells, enhancement of pro-inflammatory and profibrogenic cytokines, activation of proteolytic enzymes, defective autophagy, apoptosis and oxidative stress (Shin et al., 2013). It will be mentioned three types of LGMD as models of the possible oxidative stress involvement in their pathogenesis.

Among these muscle disorders, calpainopathy or LGMD2A is the most common form of autosomal recessive LGMD, due to the absence or reduction of the enzyme calpain 3, a non lysosomal calcium dependent cysteine protease. However, the function of calpain 3 in the muscle and the pathomechanisms of LGMD2A are not to date fully defined. Previous studies in calpainopathy mice have documented that oxidative stress occurs in LGMD2A, with the involvement of NF-kB pathway. Oxidative stress and NF-kB/IKK $b$ signaling were also observed increase in muscle of LGMD2A patients, as potential contributor to protein ubiquitinylation and 
muscle protein loss (Rajakumar et al., 2013). NF-xB and the ubiquitin pathways were also studied in muscle of patients affected by an other common form of recessive LGMD, the LGMD2B, due to dysferlin mutations, and in dysferlin knockdown human myoblasts and myotubes (Rajakumar et al., 2014). It was observed that in response to oxidative stress dysferlinopathic muscle biopsies showed protein ubiquitinylation induced by NF- $x \mathrm{~B}$ p65 signaling. Moreover, investigations on dysferlin knockdown primary muscle cell cultures indicated that oxidative stress was induced in the absence of dysferlin. At muscle level, dysferlin is involved in plasma membrane repair, vesicle fusion and membrane trafficking. Subjects with mutations in the dysferlin gene can have impaired membrane resealing after mechanical or chemical stimuli, with consequent influx of $\mathrm{Ca}^{2+}$. Since mitochondria are responsible for $\mathrm{Ca}^{2+}$ buffering, mitochondrial defects with respiratory chain deficiency in muscle from patients with dysferlinopathies has been recently documented (Vincent et al., 2016).

Another form of autosomal dominat LGMD is the type $1 \mathrm{~B}$, due to mutations in LMNA gene that encodes lamin $\mathrm{A} / \mathrm{C}$, located at the nuclear envelope. In fibroblasts, myoblasts and muscle tissue of LGMD1 patients nuclear abnormalities are well documented, consisting of alteration of nuclear lamina and loss of heterochromatin. There are evidence points to a relationship between LMNA mutation and altered redox homeostasis. In fact, a dysfunctional lamina can modulate ROS metabolism, since the nuclear envelope is a docking site for transcription factors and chromatin-associated proteins. Moreover, ROS can damage the nuclear envelope, contributing to cellular senescence and vulnerability to oxidative stress. The effects of ROS consist of steady DNA damage and telomere shortening, that are the mechanisms also involved in pathogenesis of laminopathies (Lattanzi et al., 2012; Sieprath et al., 2015).

\section{Role of physical exercise in MDs}

In the past, a common opinion was that, in subjects with muscle wasting, physical activity can accelerate muscle degeneration mechanisms. Repeated muscle contractions can reduce the strength limiting exercise performance which could result in muscle fatigue mainly due to lactic acidosis, as observed in MDs
(Allen et al., 2008). However, other reports have highlighted that in humans undergoing exercise at different intensities, lactic acid does not have deleterious effects and does not induce muscle fatigue (Reid, 2008). Muscle fibers generate ROS at low levels that increase during exercise inducing muscle fatigue (Kuwahara et al., 2010) and increasing muscle atrophy (Steinbacher and Eckl, 2015). Many other mechanisms are implicate in muscle fatigue like changes in $\mathrm{Ca}^{2+}$ homeostasis, disruption of creatine phosphate as consequence of increased levels of inorganic phosphate ions, depletion of inter- and intramyofibrillar glycogen and increased levels of ROS in the myoplasm (Allen et al., 2008). Nevertheless, considering that to date there is no curative treatment for most MDs, exercise training as treatment for these diseases, is receiving much attention. It follows from the evidence that the exercise conducted properly can help to maintain and improve health reducing the risk of several disorders (Dahlqvist and Vissing, 2016). However, dystrophic muscle fibers are known to be more susceptible to oxidative muscle damage after exercise, hence the need to understand how the muscle cells adapt to changes during physical activity, and if muscle from dystrophic patients are subjected to different coping mechanisms than those observed in control muscles (Schill et al., 2016).

\section{Oxidative stress and physical exercise}

Acute bouts of contractile exercises induced, in animal models, an increase of lipid hydroperoxides, 4-HNE, protein carbonyls levels and a reduction in total glutathione levels, as well as increased XO activity (Judge and Dodd, 2003). Interestingly, well-trained humans and rats may be resistant to sudden increases of ROS levels caused by acute and strenuous exercise (Pingitore et al., 2015). In a study conducted by Oztasan and collaborators (Oztasan et al., 2004), acute exhausting exercise decreased erythrocyte MDA levels, and increased the erythrocyte SOD activity in trained $(n=28)$ rats with respect to sedentary $(n=26)$ rats. On the other hand, the erythrocyte GPX activity was increased in sedentary rats, thought, trained rats showed no variations in the enzyme activity.

Çakır-Atabek et al. (2015) found that that lower exercise intensity $(50 \%)$ was sufficient to increase MDA levels, while only higher intensity (more 
than $80 \%$ ) was needed to induce an increase in carbonyl protein levels in trained $(\mathrm{N}=8)$ and untrained $(\mathrm{N}=8)$ men. MDA, protein carbonyl, and SOD concentrations significantly increased during exercise in trained, though MDA levels decreased during recovery and $24 \mathrm{~h}$ post-exercise. Instead, 8-OHdG and GSH values did not significantly change during the test, while GSH levels were higher in trained men with respect to sedentary subjects. The beneficial effects of regular and nonexhaustive physical activity are well known. Trained persons show higher levels of adaptation and less health risks. During regular exercise, ROS increase the antioxidant activity, mitochondrial biogenesis, cytoprotection and aerobic capacity of skeletal muscle (Steinbacher and Eckl, 2015). Physical activity promotes specific adaptations in relation to type and intensity of exercise performed, to defend the body from an excessive ROS production, improving motor performance (Castrogiovanni and Imbesi, 2012). Aerobic and regular training exerts beneficial effects decreasing oxidative damage and up-regulating antioxidant pathways (Allen et al. 2008, Falone et al., 2010; Farinha et al., 2015). So, long term, regular and moderate exercise promotes a more reducing environment, while intensive exercise training leads to more oxidizing environment (SeifiSkishahr et al., 2016).

Besides sport with both aerobic and anaerobic components, such as volleyball, may provide adequate protection against exercise-induced oxidative stress (Kocabaş et al., 2016). Kocabaş and collaborators (2016) noted, in thirteen male volleyball players, that serum total oxidant status (TOS) levels and oxidative stress index (OSI) were significantly lower after volleyball match.

\section{Physical activity in DMD and BMD}

There are evidences from $m d x$ mouse models that eccentric exercise can be more damaging to dystrophic muscle than to normal muscle (Ensrud and Kissel, 2015). Stretch-induced muscle damage is considerably more severe in the $m d x$ mouse and transfection of dystrophin into $m d x$ muscle causes a reduction of the damage. Furthermore, eccentric exercise causes a substantial increase in muscle $\mathrm{Ca}^{2+}$ which accumulates in the mitochondria resulting in activation of PLA2 and ROS production that increases lipid peroxidation and changes membrane permeability in $m d x$ mouse (Allen et al., 2008). Schill et al. (2016) have been tested in $m d x$ mice, with respect to sedentary $m d x$ and wild-type mice, if a forced treadmill exercise program (twice-weekly, for 4 weeks) influences oxidative stress and exercise capacity. They observed a lower exercise capacity in sedentary $m d x$ versus wild-type mice. After the exercise program, $m d x$ mice showed lower basal oxygen consumption and exercise capacity, but a similar maximal oxygen consumption with respect to wild-type mice. The clinical signs of pathology were increased by raising limb muscle damage and reducing exercise capacity. In addition, the skeletal muscles from trained $m d x$ mice displayed an increase of oxidative stress with higher amounts of oxidized GSH and hydroxyproline, with respect to $m d x$ sedentary mice.

In contrast, several evidences show that moderate and low intensity training (LIT) can decrease onset of motor deficits compared with minimal and excessive activity (Ensrud and Kissel, 2015). Comparing the effect of LIT (carried out by a motorized treadmill) on oxidative stress biomarkers in skeletal muscle from $m d x$ and wild-type mice, Kackzor and coworkers (2007) found higher MDA levels in white muscle from sedentary $m d x$ mice versus sedentary and LIT wild-type groups; protein carbonyl content was higher in white and red muscle of $m d x$ with respect to wild-type mice. However, antioxidant SOD, CAT and GPX activities were higher in white muscle of $m d x$ than in wild-type mice. After LIT, white muscle of $m d x$ mice showed lower MDA and protein carbonyl levels and it did not enhanced antioxidant activity of tested enzymes. Subsequently, these results have been confirmed by two independent working groups (Fontana et al., 2015; Hyzewicz et al., 2015). In the first report, Fontana et al. (2015) shown a significant recovery of damaged skeletal muscle of $m d x$ mouse following LIT (30 days). In this study, however, protein levels of SOD1 were down-regulated while carbonic anhydrase 3 levels (a member of the zinc metalloenzymes family) were up-regulated in quadriceps of sedentary $m d x$ mice. In addition, in exercised $m d x$ mice both values were significantly restored to the values of sedentary and exercised wild-type mice. Hyzevicz and collaborators (2015) found that LIT (swim training: $30 \mathrm{~min} /$ day, for 4 days/week, for one month) induced a reduction of protein carbonyls and an increase in the expression of proteins involved in mitochondria function, muscle 
contraction, glycogen metabolism, and glycolysis. Interestingly, the authors also noted that the beneficial effects of LIT were more pronounced in $m d x$ than in wild-type muscle. In particular, while in $m d x$ muscle, exercise reduced protein carbonyls levels and increased their expression, in wild-type mice LIT increased protein carbonylation but had limited influence on their expression. In a human randomized controlled trial, thirty boys with DMD were recruited and divided into two groups: intervention and control group. The first group was subjected to training of the legs (by bicycle) and arms during 24 weeks, five times per week. The second group received the same treatment after a waiting period of 24 weeks. The training was feasible and safe for both ambulant and wheelchairdependent children. It has been demonstrated that the interventions improved muscle strength in half of the patients evaluated by manual muscle testing and delayed functional deterioration (Jansen et al., 2013). Another study that investigated in adult patients with BMD the effect of aerobic and moderate-intensity training, concluded that it was an effective and safe method to increase fitness. These beneficial effects were experienced both after 12 weeks and 1 year of training highlighting the importance of rehabilitation exercises in this patients (Sven et al., 2008).

These results are important for introduce therapeutic exercise in patients with MyD in which exercise prescription remains under discussion (Hyzewicz et al., 2015).

\section{Physical activity in FSHD}

Even in FSHD aerobic exercise appears to be safe and potentially beneficial improving exercise performance (Olsen et al., 2005). Andersen et al. (2015) assessed the effect of regular aerobic training (36 sessions of 30 minute of cycle-ergometer training, for 12 weeks) in FSHD patients on fitness, walking speed, muscle strength, and daily activity levels. The authors observed that fitness, workload, physical capacity, walking speed, and health improved $(10 \%, 18 \%, 7 \%$, respectively), while muscle strength and daily activity levels did not change after the training session.

A randomized controlled trial was conducted to investigate the effects of a 24-week adapted home-based exercise training program in 19 FSHD patients $(\mathrm{N}=10$ training group, $\mathrm{N}=9$ control group, no-training) evaluating the safety and efficacy of combined aerobic, high-intensity interval and strength training on motor function, muscle histological, biochemical characteristics and quality of life. The training increased $\mathrm{VO}_{2 \max }$, maximal aerobic power, citrate synthase enzyme activity, muscle strength and endurance improved motor function (walking speed), and reduced experienced fatigue. The training intervention did not alter quality of life and muscle integrity (serum CK levels and muscle morphology). These findings suggested that combined training is a well-tolerated, safe, and effective long-term method to induce functional gains without muscle damage in FSHD patients (Bankolé et al., 2016).

Despite these evidence, another FSHD randomized controlled trial reported no aerobic power improvement after 16 weeks of aerobic training, though reduced chronic fatigue. This study was conducted on 28 FSHD patients and the aerobic training consisted of 3 weekly sessions of cycling exercise for 30 minutes (Voet et al., 2014).

Our preliminary and unpublished data on 8 FSHD patients affected by a mild-moderate form of disease show that an exercise protocol on cycle-ergometer evaluation (basal, 70\% of maximal voluntary contraction and recovery) may be easily deployable and suitable for a proper clinical assessment of fatigue in patients. Data about oxidative stress do not shown significant differences in some analyzed oxidative stress biomarkers (AOPP, FRAP and total thiols) between patients and controls $(\mathrm{N}=8)$; the levels of oxidative stress biomarkers remained stable during and after the exercise protocol, both in patients and controls, even if we observed a reduction (not significant) of oxidative damage to proteins after exercise, in FSHD patients. However, only a few subjects were analyzed and a single bouts of exercise do not allow to evaluate significant changes in oxidative stress biomarkers. There is the need to increase the number of subjects for analysis and to test if oxidative parameters can be changed in response of exercise training.

\section{Physical activity in LGMD}

As reviewed by Siciliano and collaborators, several studies have shown that exercise can be safe and beneficial also for LGMD patients (Siciliano et al., 2015). For example, Sveen et al. (2007) analyzed the effect of low intensity aerobic training in patients $(\mathrm{N}=9$ ) with LGMD2I, caused by mutations in the 
fukutin-related protein gene (FKRP). The training program consisted in 30 minute of training (fifty sessions) for 12 weeks on cycle-ergometer at $65 \%$ of $\mathrm{VO}_{2 \max }$. The different parameters were evaluated from 24 to 48 hours after the final training session, after which $\mathrm{VO}_{2 \max }$ and maximal workload were improved. Plasma lactate levels during the different exercise test steps did not differ significantly before and after 12 weeks of training and plasma CK levels increased after training in patients similarly to controls $(\mathrm{N}=9)$. Subsequently, the authors presented the results of two pilot studies on the effect of resistance training. In particular, in one study they assessed the effect of low-intensity strength training in LGMD2A $(\mathrm{N}=2)$, and LGMD2I $(\mathrm{N}=4)$ patients showing that elbow flexion and knee extension significantly increased muscle strength and endurance. In the second study, the authors investigated the effect of highintensity strength training in patients with LGMD2A $(\mathrm{N}=4)$, and LGMD2I $(\mathrm{N}=2)$. The preliminary results indicated that 3 months of resistance training could be beneficial increasing muscle strength and endurance in these patients (Sveen et al., 2013).

In another study, patients ( $\mathrm{N}=6$ ) with LGMD2L, also known as anoctaminopathy due to recessive ANO5 gene mutations, were selected for test any effects of home-based, pulse-watch monitored, moderateintensity exercise on a cycle-ergometer (30 minutes, 3 times weekly, for 10 weeks. Outcome measures were $\mathrm{VO}_{2 \max }$, performed at $70 \%$ of the $\mathrm{VO}_{2 \max }$, and time in the 5-repetitions-sit-to-stand test (FRSTST), requiring patients to stand up and sit from a chair 5 times as rapidly as possible. The authors observed a significant improvement of the $\mathrm{VO}_{2 \max }$ and FRSTST, increased oxidative capacity and muscle function, no change in the $\mathrm{CK}$ levels and no detrimental effects (Vissing et al., 2014).

\section{Physical activity in DM1}

Aldehag et al. (2013) performed a randomized controlled trial with a crossover design to investigate the effects of a training program (12 weeks) on hand-grip, testing pinch and wrist force, manual dexterity and activities of daily living, in 35 adults with DM1. The authors observed that this type of training improved wrist flexor force as well as self-perception and satisfaction of performance. No evident detrimental effects were shown, suggesting that resistance training of hand muscles can be a good therapy option for the clinical practice in DM1 patients.

Another study indicated that 12 weeks of lowintensity, aerobic training on a cycle-ergometer was an effective and safe method to improve oxidative capacity and fitness in patients with DM1 $(\mathrm{N}=12)$. The training increased $\mathrm{VO}_{2 \max }$ by $14 \%$, and maximal workload by $11 \%$, whereas CK levels remained unchanged. Moreover, muscle fiber diameter for type I and IIa fibers increased significantly and selfreported changes in activities of daily living improved during the training weeks (Orngreen et al., 2008).

Tramonti and coworkers (2014) evaluated the oxidative metabolism efficiency in 18 DM1 patients versus 15 healthy subjects, evaluating lactate levels at rest and after a submaximal incremental exercise test performed on a treadmill. The results showed, after exercise, an early induction of fatigue in patients compared to controls; moreover, patients detected normal lactate values at rest, which increased during recovery. These findings suggest an early induction of anaerobic metabolism with consequent alteration of oxidative metabolism in DM1. Considering that aerobic training improve muscle oxidative capacity and facilitates the lactate removal, it could be used to help DM1 patients with rehabilitation programs focused to limit muscle damage.

\section{Conclusions}

Despite the evidence that exercise increase the reactive species production, habitual and moderate physical activity reduces the incidence of many oxidative stress-based diseases and is advantageous also in patients with MDs. However, there is the need to conduct a systematic search to point out the effects of physical exercise in experimental settings. The identification of strategies for limiting the excess of ROS both in basal condition and during physical activity could be helpful to limit muscle damage and improve muscle function in MDs.

\section{Acknowledgements}

The authors acknowledge the support from AFMTelethon, grant number 17191, and Progetti di Ricerca d'Ateneo (PRA), University of Pisa, grant number PRA_2016_43. 


\section{References}

Aldehag A., Jonsson H., Lindblad J., Kottorp A., Ansved T., Kierkegaard M. Effects of handtraining in persons with myotonic dystrophy type 1-a randomized controlled cross-over pilot study. Disabil Rehabil., 35: 1798-807, 2013.

Allen D.G., Lamb G.D., Westerblad H. Skeletal muscle fatigue: cellular mechanisms. Physiol Rev., 88: 287-332, 2008.

Andersen G., Prahm K.P., Dahlqvist J.R., Citirak G., Vissing J. Aerobic training and postexercise protein in facioscapulohumeral muscular dystrophy: RCT study. Neurology. 85: 396-403, 2015.

Bankolé L.C., Millet G.Y., Temesi J., Bachasson D., Ravelojaona M., Wuyam B., Verges S., Ponsot E., Antoine J.C., Kadi F., Féasson L. Safety and efficacy of a 6-month home-based exercise program in patients with facioscapulohumeral muscular dystrophy: A randomized controlled trial. Medicine (Baltimore). 95: e4497, 2016.

Boccatonda A., Tripaldi R., Davì G., Santilli F. Oxidative Stress Modulation Through Habitual Physical Activity. Curr Pharm Des., 22: 3648-80, 2016.

Bouzid M.A., Filaire E., McCall A., Fabre C. Radical Oxygen Species, Exercise and Aging: An Update. Sports Med., 45: 1245-61, 2015.

Brand M.D. The sites and topology of mitochondrial superoxide production. Exp Gerontol., 45: 466-72, 2010.

Çakır-Atabek H., Özdemir F., Çolak R. Oxidative stress and antioxidant responses to progressive resistance exercise intensity in trained and untrained males. Biol Sport., 32: 321-8, 2015.

Castrogiovanni P., Imbesi R. Oxidative stress and skeletal muscle in exercise. Ital J Anat Embryol., 117: 107-17, 2012.

Chalimoniuk M., Chrapusta S.J., Lukačova N., Langfort J. Endurance training upregulates the nitric oxide/soluble guanylyl cyclase/cyclic guanosine 3', 5'-monophosphate pathway in the striatum, midbrain and cerebellum of male rats. Brain Res., 1618: 29-40, 2015.

Choi M.H., Ow J.R., Yang N.D., Taneja R. Oxidative Stress-Mediated Skeletal Muscle Degeneration: Molecules, Mechanisms, and Therapies. Oxid Med Cell Longev., 2016.

Chung H.Y., Baek B.S., Song S.H., Kim M.S., Huh J.I., Shim K.H., Kim K.W., Lee K.H. Xanthine dehydrogenase/xanthine oxidase and oxidative stress. Age (Omaha), 20: 127-40, 1997.

Cipak Gasparovic A., Zarkovic N., Zarkovic K., Semen K., Kaminskyy D., Yelisyeyeva O., Bottari
S.P. Biomarkers of oxidative and nitro-oxidative stress: conventional and novel approaches. $\mathrm{Br} J$ Pharmacol., 2016.

Dahlqvist J.R., Vissing J. Exercise Therapy in Spinobulbar Muscular Atrophy and Other Neuromuscular Disorders. J Mol Neurosci., 58: 388-93, 2016.

Darras B.T.,MillerD.T.,UrionD.K.Dystrophinopathies. [updated 2014 Nov 26], 2000. In: Pagon R.A., Adam M.P., Ardinger H.H., Wallace S.E., Amemiya A., Bean L.J.H., Bird T.D., Fong C.T., Mefford H.C., Smith R.J.H., Stephens K., editors. GeneReviews® [Internet]. Seattle (WA): University of Washington Seattle, 1993-2016.

Davies K.E., Nowak K.J. Molecular mechanisms of muscular dystrophies: old and new players. Nature Reviews Molecular Cell Biology. 7: 762-773, 2006.

Dmitriev P., Bou Saada Y., Dib C., Ansseau E., Barat A., Hamade A., Dessen P., Robert T., Lazar V., Louzada R.A., Dupuy C., Zakharova V., Carnac G., Lipinski M., Vassetzky Y.S. DUX4-induced constitutive DNA damage and oxidative stress contribute to aberrant differentiation of myoblasts from FSHD patients. Free Radic Biol Med., 99: 244-258, 2016.

Ensrud E., Kissel J.T. Aerobic exercise in muscular dystrophy: gain without pain. Neurology. 85: 3923,2015 .

Ervasti J.M., Campbell K.P. Membrane organization of the dystrophin-glycoprotein complex. Cell, 66: 1121-1131, 1991.

Falone S., Mirabilio A., Pennelli A., Cacchio M., Di Baldassarre A., Gallina S., Passerini A., Amicarelli F. Differential impact of acute bout of exercise on redox- and oxidative damage-related profiles between untrained subjects and amateur runners. Physiol Res., 59: 953-61, 2010.

Farinha J.B., Steckling F.M., Stefanello S.T., Cardoso M.S., Nunes L.S., Barcelos R.P., Duarte T., Kretzmann N.A., Mota C.B., Bresciani G., Moresco R.N., Duarte M.M., Dos Santos D.L., Soares F.A. Response of oxidative stress and inflammatory biomarkers to a 12-week aerobic exercise training in women with metabolic syndrome. Sports Med Open., 1: 3, 2015.

Fisher-Wellman K., Bloomer R.J. Acute exercise and oxidative stress: a 30 year history. Dyn Med., 8: 1, 2009.

Fontana S., Schillaci O., Frinchi M., Giallombardo M., Morici G., Di Liberto V., Alessandro R., De Leo G., Perciavalle V., Belluardo N., Mudò G. Reduction in mdx mouse muscle degeneration by low-intensity endurance exercise: a protomi analysis in quadriceps 
muscle of exercised compared with sedentary mdx mice. Biosci Rep., 35: pii: e00213, 2015.

Frijhoff J., Winyard P.G., Zarkovic N., Davies S.S., Stocker R., Cheng D., Knight A.R., Taylor E.L., Oettrich J., Ruskovska T., Gasparovic A.C., Cuadrado A., Weber D., Poulsen H.E., Grune T., Schmidt H.H., Ghezzi P. Clinical Relevance of Biomarkers of Oxidative Stress. Antioxid Redox Signal., 23: 1144-70, 2015.

Gatica L.V., Rosa A.L. A complex interplay of genetic and epigenetic events leads to abnormal expression of the DUX4 gene in facioscapulohumeral muscular dystrophy. Neuromuscul Disord., 26: 844-852, 2016.

Giorgio M., Trinei M., Migliaccio E., Pelicci P.G. Hydrogen peroxide: a metabolic by-product or a common mediator of ageing signals? Nat Rev Mol Cell Biol., 8: 722-8, 2007.

Gomez-Cabrera M.C., Domenech E., Viña J. Moderate exercise is an antioxidant: upregulation of antioxidant genes by training. Free Radic Biol Med., 44: 126-31, 2008.

Goncalves R.L., Quinlan C.L., Perevoshchikova I.V., Hey-Mogensen M., Brand M.D. Sites of superoxide and hydrogen peroxide production by muscle mitochondria assessed ex vivo under conditions mimicking rest and exercise. $J$ Biol Chem., 290: 209-27, 2015.

Grozdanovic Z., Christova T., Gosztonyi G., Mellerowicz H., Blottner D., Grossran R. Absence of nitric oxide synthase I despite the presence of the dystrophin complex in human striated muscle. Histochem J., 97-104, 1997.

Gücüyener K., Ergenekon E., Erbas D., Pinarli G., Serdaroğlu A. The serum nitric oxide levels in patients with Duchenne muscular dystrophy. Brain Dev., 22: 181-3, 2000.

Halliwell B. Biochemistry of oxidative stress. Biochem Soc Trans., 35: 1147-50, 2007.

He F., Li J., Liu Z., Chuang C.C., Yang W., Zuo L. Redox Mechanism of Reactive Oxygen Species in Exercise. Front Physiol., 7: 486, 2016.

Heydemann A., McNally E. NO more muscle fatigue. J Clin Invest., 119: 448-50, 2009.

Hyzewicz J., Tanihata J., Kuraoka M., Ito N., Miyagoe-Suzuki Y., Takeda S. Low intensity training of mdx mice reduces carbonylation and increases expression levels of proteins involved in energy metabolism and muscle contraction. Free Radic Biol Med., 82: 122-36, 2015.

Jansen M., van Alfen N., Geurts AC., de Groot I.J. Assisted bicycle training delays functional deterioration in boys with Duchenne muscular dystrophy: the randomized controlled trial "no use is disuse". Neurorehabil Neural Repair., 27: 81627, 2013.

Judge A.R., Dodd S.L. Oxidative damage to skeletal muscle following an acute bout of contractile claudication. Atherosclerosis, 171: 219-24, 2003.

Kaczor J.J., Hall J.E., Payne E., Tarnopolsky M.A. Low intensity training decreases markers of oxidative stress in skeletal muscle of mdx mice. Free Radic Biol Med., 43: 145-54, 2007.

Kar N.C., Pearson C.M. Catalase superoxide dismutase glutathione reductase and thiobarbituric acid-reactive products in normal and dystrophic human muscle. Clin Chim Acta., 94: 277-80, 1979.

Kocabaş R., Namiduru E.S., Bagçeci A.M., Erenler A.K., Karakoç Ö., Örkmez M., Akan M., Erdemli H.K., Taysi S., Tarakçioglu M. The acute effects of interval exercise on oxidative stress and antioxidant status in volleyball players. $J$ Sports Med Phys Fitness. [Epub ahead of print], 2016.

Kumar A., Kumar V., Singh S.K., Muthuswamy S., Agarwal S. Imbalanced oxidant and antioxidant ratio in myotonic dystrophy type 1 . Free Radic Res., 48: 503-10, 2014.

Kurutas E.B. The importance of antioxidants which play the role in cellular response against oxidative/ nitrosative stress: current state. Nutr J., 15: 71, 2016.

Kuwahara H., Horie T., Ishikawa S., Tsuda C., Kawakami S., Noda Y., Kaneko T., Tahara S., Tachibana T., Okabe M., Melki J., Takano R., Toda T., Morikawa D., Nojiri H., Kurosawa H., Shirasawa T., Shimizu T. Oxidative stress in skeletal muscle causes severe disturbance of exercise activity without muscle atrophy. Free Radic Biol Med., 48: 1252-62, 2010.

Lamb G.D., Westerblad H. Acute effects of reactive oxygen and nitrogen species on the contractile function of skeletal muscle. J Physiol., 589: 211927, 2011.

Lattanzi G., Marmiroli S., Facchini A., Maraldi N.M. Nuclear damages and oxidative stress: new perspectives for laminopathies. Eur J Histochem. 56: e45, 2012. doi: 10.4081/ejh.2012.e45.

Lemmers R.J., Goeman J.J., van der Vliet P.J., van Nieuwenhuizen M.P., Balog J., Vos-Versteeg M., Camano P., Ramos Arroyo M.A., Jerico I., Rogers M.T., Miller D.G., Upadhyaya M., Verschuuren J.J., Lopez de Munain Arregui A., van Engelen B.G., Padberg G.W., Sacconi S., Tawil R., Tapscott S.J., Bakker B., van der Maarel S.M. Inter-individual differences in $\mathrm{CpG}$ methylation at 
D4Z4 correlate with clinical variability in FSHD1 and FSHD2. Hum Mol Genet., 24: 659-69, 2015.

Mangge H., Becker K., Fuchs D., Gostner J.M. Antioxidants, inflammation and cardiovascular disease. World J Cardiol., 6: 462-77, 2014.

Nikolić-Kokić A., Marinković D., Perić S., Stević Z., Spasić M.B., Blagojević D., Rakoc`ević-Stojanović $\mathrm{V}$. Redox imbalance in peripheral blood of type 1 myotonic dystrophy patients. Redox Rep., 21: 2327, 2016.

Olsen D.B., Ørngreen M.C., Vissing J. Aerobic training improves exercise performance in facioscapulohumeral muscular dystrophy. Neurology. 64: 1064-6, 2005.

Orngreen M.C., Olsen D.B., Vissing J. Aerobic training in patients with myotonic dystrophy type 1. Ann Neurol. 200557 754-7. PubMed PMID: 15852373.

Oztasan N., Taysi S., Gumustekin K., Altinkaynak K., Aktas O., Timur H., Siktar E., Keles S., Akar S., Akcay F., Dane S., Gul M. Endurance training attenuates exercise-induced oxidative stress in erythrocytes in rat. Eur J Appl Physiol., 91: 622-7, 2004.

Pacher P., Beckman J.S., Liaudet L. Nitric oxide and peroxynitrite in health and disease. Physiol Rev., 87: 315-424, 2007.

Pantic B., Trevisan E., Citta A., Rigobello M.P., Marin O., Bernardi P., Salvatori S., Rasola A. Myotonic dystrophy protein kinase (DMPK) prevents ROS-induced cell death by assembling a hexokinase II-Src complex on the mitochondrial surface. Cell Death Dis., 4: e858, 2013.

Pendyala S., Natarajan V. Redox regulation of Nox proteins. Respir Physiol Neurobiol., 174: 265-71, 2010.

Pingitore A., Lima G.P., Mastorci F., Quinones A., Iervasi G., Vassalle C. Exercise and oxidative stress: potential effects of antioxidant dietary strategies in sports. Nutrition., 31: 916-22, 2015.

Pisoschi A.M., Pop A. The role of antioxidants in the chemistry of oxidative stress: A review. Eur J Med Chem., 97: 55-74, 2015.

Powers S.K., Jackson M.J. Exercise-induced oxidative stress: cellular mechanisms and impact on muscle force production. Physiol Rev., 88: 1243-76, 2008.

Ragusa R.J., Chow C.K., Porter J.D. Oxidative stress as a potential pathogenic mechanism in an animal model of Duchenne muscular dystrophy. Neuromuscul Disord., 7: 379-86, 1997.

Rajakumar D., Alexander M., Oommen A. Oxidative stress $\mathrm{NF}-x \mathrm{~B}$ and the ubiquitin proteasomal pathway in the pathology of calpainopathy. Neurochem Res. 38: 2009-18, 2013.

Rajakumar D., Senguttuvan S., Alexander M., Oommen A. Involvement of oxidative stress nuclear factor kappa B and the ubiquitin proteasomal pathway in dysferlinopathy. Life Sci., 108: 54-61, 2014.

Rando T.A. Role of nitric oxide in the pathogenesis of muscular dystrophies: a 'two hit' hypothesis of the cause of muscle necrosis. Microscopy Research and Technique. 55: 223-235, 2001.

Rando T.A., Disatnik M.H., Yu Y., Franco A. Muscle cells from mdx mice have an increased susceptibility to oxidative stress. Neuromuscul Disord., 8: 14-21, 1998.

Reid M.B. Free radicals and muscle fatigue: Of ROS, canaries, and the IOC. Free Radic Biol Med., 44: 169-79, 2008.

Ricci G., Scionti I., Sera F., Govi M., D’Amico R., Frambolli I., Mele F., Filosto M., Vercelli L., Ruggiero L., Berardinelli A., Angelini C., Antonini G., Bucci E., Cao M., Daolio J., Di Muzio A., Di Leo R., Galluzzi G., Iannaccone E., Maggi L., Maruotti V., Moggio M., Mongini T., Morandi L., Nikolic A., Pastorello E., Ricci E., Rodolico C., Santoro L., Servida M., Siciliano G., Tomelleri G., Tupler R. Large scale genotype-phenotype analyses indicate that novel prognostic tools are required for families with facioscapulohumeral muscular dystrophy. Brain. 136: 3408-17, 2013.

Ricci G., Zatz M., Tupler R. Facioscapulohumeral muscular dystrophy: more complex than it appears. Curr Mol Med., 2014. [Epub ahead of print].

Rodriguez M.C., Tarnopolsky M.A. Patients with dystrophinopathy show evidence of increased oxidative stress. Free Radic Biol Med., 34: 1217-20, 2003.

Ryan M.J., Jackson J.R., Hao Y., Leonard S.S., Always S.E. Inhibition of xanthine oxidase reduces oxidative stress and improves skeletal muscle function in response to electrically stimulated isometric contractions in aged mice. Free Radic Biol Med., 51: 38-52, 2011.

Schieber M., Chande N.S. ROS function in redox signaling and oxidative stress. Curr Biol., 24: R453-62, 2014.

Schill K.E., Altenberger A.R., Lowe J., Periasamy M., Villamena F.A., Rafael-Fortney J.A., Devor S.T. Muscle damage metabolism and oxidative stress in mdx mice: Impact of aerobic running. Muscle Nerve. 54: 110-7, 2016.

Seifi-Skishahr F., Damirchi A., Farjaminezhad M., Babaei P. Physical Training Status Determines 
Oxidative Stress and Redox Changes in Response to an Acute Aerobic Exercise. Biochem Res Int., 3757623, 2016.

Selsby T.J. "Increased catalase expression improves muscle function in $\mathrm{mdx}$ mice" Experimental Physiology. 96: 194-202, 2011.

Shin J., Tajrishi M.M., Ogura Y., Kumar A. Wasting mechanisms in muscular dystrophy. Int J Biochem Cell Biol., 45: 2266-79, 2013.

Siciliano G., Pasquali L., Rocchi A., Falorni M., Galluzzi F., Rocco A., Malvaldi G., Pompella A., Paolicchi A. Advanced oxidation protein products in serum of patients with myotonic disease type I: association with serum gammaglutamyltransferase and disease severity. Clin Chem Lab Med., 43: 745-7, 2005.

Siciliano G., Simoncini C., Giannotti S., Zampa V., Angelini C., Ricci G. Muscle exercise in limb girdle muscular dystrophies: pitfall and advantages. Acta Myol., 34: 3-8, 2015.

Sieprath T., Corne T.D., Nooteboom M., Grootaert C., Rajkovic A., Buysschaert B., Robijns J., Broers J.L. Ramaekers F.C., Koopman W.J., Willems P.H., De Vos W.H. Sustained accumulation of prelamin A and depletion of lamin A/C both cause oxidative stress and mitochondrial dysfunction but induce different cell fates. Nucleus. 6: 236-46, 2015.

Stamler J.S., Meissner G. Physiology of nitric oxide in skeletal muscle. Physiol Rev., 81: 209-237, 2001.

Steinbacher P., Eckl P. Impact of oxidative stress on exercising skeletal muscle. Biomolecules, 5: 35677, 2015.

Sveen M.L., Andersen S.P., Ingelsrud L.H., Blichter S., Olsen N.E., Jønck S., Krag T.O., Vissing J. Resistance training in patients with limb-girdle and becker muscular dystrophies. Muscle Nerve. 47: 163-9, 2013.

Sveen M.L., Jeppesen T.D., Hauerslev S., Køber L., Krag T.O., Vissing J. Endurance training improves fitness and strength in patients with Becker muscular dystrophy. Brain. 131: 2824-31, 2008.

Sveen M.L., Jeppesen T.D., Hauerslev S., Krag T.O., Vissing J. Endurance training: an effective and safe treatment for patients with LGMD2I. Neurology. 68: 59-61, 2007.
Tidball J.G., Wehling-Henricks M. Expression of a NOS transgene in dystrophin-deficient muscle reduces muscle membrane damage without increasing the expression of membrane-associated cytoskeletal proteins. Mol Genet Metab., 82: 312-20, 2004.

Tramonti C., Dalise S., Bertolucci F., Rossi B., Chisari C. Abnormal Lactate Levels Affect Motor Performance in Myotonic Dystrophy Type 1. Eur J Transl Myol., 24: 4726, 2014.

Turki A., Hayot M., Carnac G., Pillard F., Passerieux E., Bommart S., Raynaud de Mauverger E., Hugon G., Pincemail J., Pietri S., Lambert K., Belayew A., Vassetzky Y., Juntas Morales R., Mercier J., LaoudjChenivesse D. Functional muscle impairment in facioscapulohumeral muscular dystrophy is correlated with oxidative stress and mitochondrial dysfunction. Free Radic Biol Med., 53: 1068-79, 2012.

Usuki F., Ishiura S. Expanded CTG repeats in myotonin protein kinase increase susceptibility to oxidative stress. Neuroreport., 9: 2291-6, 1998.

Usuki F., Takahashi N., Sasagawa N., Ishiura S. Differential signaling pathways following oxidative stress in mutant myotonin protein kinase cDNAtransfected C2C12 cell lines. Biochem Biophys Res Coтmun., 267: 739-43, 2000.

Vincent A.E., Rosa H.S., Alston C.L., Grady J.P., Rygiel K.A., Rocha M.C., Barresi R., Taylor R.W., Turnbull D.M. Dysferlin mutations and mitochondrial dysfunction. Neuromuscul Disord., 26: 782-788, 2016.

Vissing C.R., Preisler N., Husu E., Prahm K.P., Vissing J. Aerobic training in patients with anoctamin 5 myopathy and hyperckemia. Muscle Nerve. 50: 119-23, 2014.

Voet N., Bleijenberg G., Hendriks J., de Groot I., Padberg G., van Engelen B., Geurts A. Both aerobic exercise and cognitive-behavioral therapy reduce chronic fatigue in FSHD: an RCT. Neurology. 83: 1914-22, 2014.

Wang X., Robinson P.J. Cyclic GMP-dependent protein kinase and cellular signaling in the nervous system. J Neurochem., 68: 443-56, 1997.

Wehling M., Spencer M.J., Tidball J.G. A nitric oxide synthase transgene ameliorates muscular dystrophy in mdx mice. J Cell Biol., 155: 123-31, 2001.

Westerblad H., Allen D.G. Emerging roles of ROS/ RNS in muscle function and fatigue. Antioxid Redox Signal., 15: 2487-99, 2011. 